\title{
BMJ Open Associations of volume and other hospital characteristics on mortality within 30 days of acute myocardial infarction in South Korea
}

Kyu-Tae Han, ${ }^{1,2}$ Sun Jung Kim, ${ }^{3}$ Woorim Kim, ${ }^{1,2}$ Sung-In Jang, ${ }^{1,2,4}$ Ki-Bong Yoo, ${ }^{5}$ Seo Yoon Lee, ${ }^{2}$ Eun-Cheol Park ${ }^{2,4}$

To cite: Han K-T, Kim SJ, Kim W, et al. Associations of volume and other hospital characteristics on mortality within 30 days of acute myocardial infarction in South Korea. BMJ Open 2015;5:e009186. doi:10.1136/bmjopen-2015009186

- Prepublication history for this paper is available online. To view these files please visit the journal online (http://dx.doi.org/10.1136/ bmjopen-2015-009186).

Received 24 June 2015 Revised 13 October 2015 Accepted 14 October 2015

CrossMark

For numbered affiliations see end of article.

Correspondence to Dr Eun-Cheol Park; ecpark@yuhs.ac

\section{ABSTRACT}

Objective: The mortality for acute myocardial infarction (AMI) has declined worldwide. However, improvements in care for AMI in South Korea have lagged slightly behind those in other countries. Therefore, it is important to investigate how factors such as hospital volume, structural characteristics of hospital and hospital staffing level affect 30-day mortality due to AMI in South Korea.

Setting: We used health insurance claim data from 114 hospitals to analyse 30-day mortality for AMI.

Participants: These data consisted of 19638 hospitalisations during 2010-2013.

Interventions: No interventions were made.

Outcome measure: Multilevel models were analysed to examine the association between the 30-day mortality and inpatient and hospital level variables.

Results: In the 30 days after hospitalisation, $10.5 \%$ of patients with AMI died. Hospitalisation cases at hospitals with a higher AMI volume had generally inverse associations with 30-day mortality (1st quartile=ref; 2nd quartile=OR $0.811,95 \% \mathrm{Cl} 0.658$ to 0.998 , 3rd quartile $=0 \mathrm{R} 0.648,95 \% \mathrm{Cl} 0.500$ to 0.840 , 4th quartile=OR $0.807,95 \% \mathrm{Cl} 0.573$ to 1.138 ). In addition, hospitals with a greater proportion of specialists were associated with better outcomes (above median=OR $0.789,95 \% \mathrm{Cl} 0.663$ to 0.940 ).

Conclusions: Health policymakers need to include volume and staffing when defining the framework for treatment of AMI in South Korean hospitals. Otherwise, they must consider increasing the proportion of specialists or regulating the hiring of emergency medicine specialists. In conclusion, they must make an effort to reduce 30-day mortality following AMI based on such considerations.

\section{INTRODUCTION}

Acute myocardial infarction (AMI) occurs when blood flow to the heart is severely reduced or cut-off completely. AMI often strikes with no warning, as atherosclerosis, a sentinel cause of AMI, has no symptoms. ${ }^{1}$

\section{Strengths and limitations of this study}

- Our results provide the government with a potential solution for controlling outcomes in patients with acute myocardial infarction.

- Our models considered the hierarchical nature of claim data, capturing the diversity of inpatients and hospitals as much as possible.

- We only included 156 hospitals in this study, as the national health insurance claim data we used did not include data from all hospitals in South Korea due to access authority and ethical issues.

- We were unable to determine whether the same inpatients were hospitalised multiple times, as the data used in our study were based on only hospitalisation cases.

Consequently, until recently, AMI led to high mortality worldwide.

Developments in medical technology and clinician guidelines ${ }^{2}{ }^{3}$ have led to consistent declines in worldwide AMI mortality. In South Korea, improvements in care for AMI have been reported since 2007 when an initial evaluation project began. ${ }^{4}$ Nevertheless, 30-day mortality after AMI hospitalisation, which provides good indications of acute care quality, is higher in South Korea than in other Organization for Economic Co-operation and Development (OECD) countries (OECD average: 10.8 deaths/100 patients; South Korea: 11.2 deaths/100 patients). ${ }^{5}$ Moreover, ischaemic heart disease, including AMI, is still a major cause of death all over the world (7.4 million deaths in 2012, accounting for $13.2 \%$ of all deaths). ${ }^{6}$

Many studies on factors related to 30-day AMI mortality have been conducted in the past. Some have found that hospitals with higher AMI volumes have lower mortality, as volume leads to the mastery of specific 
treatments. ${ }^{7-12}$ In addition, previous studies have shown that 30-day AMI mortality is associated with patient and hospital characteristics, such as length of stay, sex, type of insurance coverage, age, hospital ownership status, etc. $^{13-19}$

To date, some studies have examined the relationship between outcomes in AMI and clinical factors as surgery and procedure, but there are a few studies which examined the relationship between 30-day AMI mortality and hospital characteristics in South Korea. Therefore, it is important to explore how factors such as hospital volume and staffing level affect 30-day mortality after AMI in South Korean hospitals. We investigated the relationship between 30-day AMI mortality and hospital volume, as well as other characteristics. In particular, we analysed the effect of the number of emergency and cardiothoracic medicine specialists present.

\section{MATERIALS AND METHODS}

\section{Data source}

The data used in this study was a proportion of the National Health Insurance (NHI) Claim data. After the introduction of the National Health Insurance Services (NHIS) in 1989, medical records of each patient could be collected by the South Korean government. These types of data included information about patients' utilisation of healthcare resources based on their needs, and these data also collected information about hospitals which each patient visited. In addition, data used in this study could consider details about death by being merged with mortality data based on personal identification codes in the national database, as well as both patient-level and hospital-level characteristics. Although it would have been ideal had we been able to use data from all hospitals in South Korea, there were some difficulties in accessing patient information due to issues such as ethics. Therefore, we needed to extract representative hospital samples from all hospitals effectively. However, in this process, there were some limitations in linking each patient and each hospitalisation case using the encrypted information because data are provided after extraction from the national database due to ethical issues, making it impossible to identify each patient afterwards.

\section{Study population}

There were about 1730 hospitals (including 39 public hospitals) in operation during 2010-2013 in South Korea. Given that the number of public hospitals was just 39 among the total 1730 hospitals in South Korea, we assumed that it could cause baseline imbalances due to differences in hospital characteristics. Thus, the propensity score matching was used to reduce bias caused by the observed covariates and to deal with the usual baseline imbalances across hospitals. ${ }^{20}$ Therefore, we included 156 hospitals (117 private and 39 public) that were determined using propensity score-matching methods (1:3 ratio of public hospitals vs private hospitals), adjusting for region, nurse staffing level, number of total beds, number of intensive care unit (ICU) beds, number of emergency room (ER) beds and number of doctors. In the propensity score-matching methods, the outcome variable is propensity score. The propensity score is the probability of a unit being assigned to a particular treatment given a set of observed covariates. On the basis of these scores, we matched each sample using the nearest neighbour methods. ${ }^{21}$ Among these 156 hospitals, we excluded those without AMI inpatient cases $(\mathrm{N}=42)$. Ultimately, 114 hospitals (31 public and 83 private; 19638 hospitalisation cases) were included for analysis. The unit of analysis was each hospitalisation rather than each patient.

\section{Variables}

The outcome variable in this study was death within 30-day of the date of each hospitalisation for AMI. The data used in our study only consisted of hospitalisation cases, although the details of the date of death were included with each case. We identified each hospitalisation's first date of admission in the calendar year during the study period as the index date. If the date of death for each hospitalisation case was included within 30 calendar days of the index date, the case was classified as mortality within 30-day. Thus, the outcome variable in this study was 30-day mortality after admission, including both in-hospital mortality and mortality after discharge.

The primary variables of interest in relation to death within 30-day of hospitalisation were hospital volume and other hospital characteristics. Before analysing the data, we first tested whether hospital variables achieved assumptions of normality or linearity, and then we decided to categorise hospital variables on the basis of the results of these tests. The number of hospitalisation cases due to AMI (ie, hospital volume) was categorised by quartile (25th quartile: 368 , 50th quartile: 605 , and $75 \%$ quartile: 1214). Hospital characteristics included structural characteristics, such as the number of beds, the number of ICU beds, the number of ER beds, teaching status, ownership status and region, as well as human resource variables, such as the proportion of specialists, the number of cardiothoracic medicine specialists and the number of emergency medicine specialists. The proportion of specialists represents the number of specialists divided by the total number of doctors, reflecting the specialised expertise present in each hospital. These variables were used after calculating mean values of each hospital during the study period (2010-2013), and categorised by the median value of each variable based on the tests for normality or linearity.

Additionally, our models included inpatient-level variables, such as major diagnosis, the Charlson Comorbidity Index, type of insurance coverage, age and year of hospitalisation. Major diagnoses were characterised according to the International Classification of Diseases groupings (ICD-10: I21.0, I21.1, I21.2, I21.3, 
I21.4, I21.9) to reflect specific pathological mechanisms. The Charlson Comorbidity Index was calculated on the basis of whether a particular condition was present, excluding the major symptom at the date of admission to reflect the effect of comorbid disorders or diseases. Type of insurance coverage was categorised as beneficiaries of NHI or Medical Aid. Most of the general population who were covered by the NHI after paying the insurance fee charged on the basis of economic status evaluation, whereas a few low-income, disabled and elderly populations were covered by Medical Aid, being offered free insurance by the government. ${ }^{22}$

\section{Statistical analysis}

We examined the distribution of each categorical variable by examining frequencies and percentages and performed $\chi^{2}$ tests to investigate the associations with 30-day mortality. These analyses were performed for inpatient-level and hospital-level variables. Next, we performed hierarchical logistic regression analysis using multilevel models with the Generalized Linear Mixed Model (GLIMMIX) procedure including inpatient-level and hospital-level variables, analysed to examine the associations with 30-day mortality after hospitalisation. Additionally, subgroup analyses for multilevel models were performed according to the median number of specialists in emergency or cardiothoracic medicine. These were also performed after stratifying on the basis of regional hospital characteristics. All statistical analyses were performed using SAS statistical software V.9.2. All $\mathrm{p}$ values calculated were two-sided and considered significant at $\mathrm{p}<0.05$.

\section{RESULTS}

The data used in this analysis consisted of 19638 hospitalisations. Of these, 17605 inpatients $(89.5 \%)$ survived, and $2033(10.5 \%)$ died within 30-day after the date of each admission. Table 1 shows the univariate associations between independent variables and 30-day mortality due to AMI. In terms of detailed major diagnoses, 'Acute transmural myocardial infarction of inferior wall (ICD-10: I21.1)' was associated with the lowest 30-day mortality rate after AMI hospitalisation (5.5\%). Lower Charlson Comorbidity Index scores were associated with higher 30-day survival rates (score $=0$ : $2.7 \%$, score $=1$ : $2.7 \%$, score $=2: 4.7 \%$, score $=3+: 15.4 \%$ ). The 30 -day mortality rate for males $(7.6 \%)$ was lower than that for females $(16.4 \%)$. Finally, inpatients of the general population, who were covered by national health insurance, had lower mortality than beneficiaries of Medical Aid, which provides free inpatient and outpatient care for the low-income, disabled and elderly populations by using government funds (NHI: 9.9\%, Medical Aid: $15.4 \%)$. In the distribution of hospital-level variables, 141 hospitals were used in this study. Regarding the results of hospital volume due to AMI, hospitals with higher AMI volumes were associated with lower 30-day mortality (1st quartile: $17.8 \%$, 2nd quartile: $8.9 \%$, 3rd quartile: $7.9 \%$, 4th quartile: $7.3 \%$; $<<0.001)$. However, hospitals scoring below the median for the proportion of specialists were shown less frequently in 30-day mortality due to AMI than those above the median (below median: $8.4 \%$, above median: $12.3 \%$ ). In terms of hospital staffing, hospitals with a higher number of cardiothoracic medicine or emergency medicine specialists were associated with lower 30-day mortality. On the basis of ownership or teaching status, private or non-teaching hospitals were shown less frequently in 30-day mortality than other types of hospitals (table 1).

Next, we performed hierarchical logistic regression analysis using a multilevel model adjusting for inpatient-level and hospital-level variables to investigate the relationships with 30-day mortality in inpatients with AMI. The results revealed that inpatients with a diagnosis of 'acute transmural myocardial infarction of unspecified sites (ICD-10: I21.4)' had the highest risk of death in the first 30-days following AMI hospitalisation compared with inpatients with AMI of the inferior wall (OR $2.451,95 \%$ CI 1.729 to 3.475 ). Hospitalisation cases with higher than three Charlson Comorbidity Index scores were also associated with a higher risk of 30-day mortality. Additionally, females had a higher risk of 30-day mortality than males. Finally, inpatients aged $\geq 65$ years also had a higher 30-day risk of death. In terms of hospitallevel variables, hospitalisation cases at hospital with a higher AMI volume had generally inverse associations with 30-day mortality (1st quartile=ref; 2 nd quartile $=\mathrm{OR}$ $0.811,95 \%$ CI 0.658 to 0.998 , 3rd quartile $=$ OR 0.648 , $95 \%$ CI 0.500 to 0.840 , 4 th quartile=OR $0.807,95 \%$ CI 0.573 to 1.138$)$. Also, hospitalisation cases at hospitals with a greater proportion of specialists were also associated with a lower mortality risk (OR 0.789 , 95\% CI 0.663 to 0.940 ). Similarly, hospitalisation cases at hospitals with more emergency medicine specialists had a lower level of 30-day mortality. With regard to structural characteristics, hospitalisation cases at teaching hospitals had a lower mortality risk than those at non-teaching hospitals. In addition, cases at private hospitals had a lower risk of 30-day following hospitalisation due to AMI. With regard to the regional characteristics of hospitals, hospitals in non-metropolitan regions had an inverse association with 30-day mortality (table 2).

We performed additional analysis for hierarchical logistic regression analysis using a multilevel model to investigate the differences in association with 30-day mortality after stratifying by the median number of specialists in emergency and cardiothoracic medicine, respectively. A similar analysis was also performed after stratifying by regional hospital characteristics. The overall trends in the subgroup analyses were similar to the results in the full model analyses. There were some differences related to hospital volume or staffing. In the results of the subgroup analysis by median value of emergency or cardiothoracic medicine, hospitals with a higher AMI volume were inversely associated with 30-day 
Table 1 Associations between inpatient or hospital characteristics and 30-day mortality after AMI hospitalisation

\begin{tabular}{|c|c|c|c|c|c|}
\hline \multirow[b]{2}{*}{ Variables } & \multicolumn{2}{|c|}{ Survived } & \multicolumn{2}{|l|}{ Died } & \multirow[b]{2}{*}{ p Value } \\
\hline & $\mathbf{N}$ & Per cent & $\mathbf{N}$ & Per cent & \\
\hline \multicolumn{6}{|l|}{ Inpatient-level variables } \\
\hline \multicolumn{6}{|l|}{ Major diagnosis } \\
\hline Acute transmural myocardial infarction of anterior wall & 1748 & 92.5 & 141 & 7.5 & $<0.0001$ \\
\hline Acute transmural myocardial infarction of inferior wall & 1451 & 94.5 & 85 & 5.5 & \\
\hline Acute transmural myocardial infarction of other sites & 212 & 88.3 & 28 & 11.7 & \\
\hline Acute transmural myocardial infarction of unspecified sites & 1836 & 91.2 & 178 & 8.8 & \\
\hline Acute subendocardial myocardial infarction & 3895 & 93.3 & 279 & 6.7 & \\
\hline Acute myocardial infarction, unspecified & 8463 & 86.5 & 1322 & 13.5 & \\
\hline \multicolumn{6}{|l|}{ Charlson Comorbidity Index } \\
\hline 0 & 1594 & 97.3 & 45 & 2.7 & $<0.0001$ \\
\hline 1 & 3134 & 97.3 & 87 & 2.7 & \\
\hline 2 & 3321 & 95.3 & 165 & 4.7 & \\
\hline $3+$ & 9556 & 84.6 & 1736 & 15.4 & \\
\hline \multicolumn{6}{|l|}{ Sex } \\
\hline Male & 12419 & 92.4 & 1018 & 7.6 & $<0.0001$ \\
\hline Female & 5186 & 83.6 & 1015 & 16.4 & \\
\hline \multicolumn{6}{|l|}{ Type of insurance coverage } \\
\hline $\mathrm{NHI}$ & 16129 & 90.1 & 1764 & 9.9 & $<0.0001$ \\
\hline Medical Aid & 1476 & 84.6 & 269 & 15.4 & \\
\hline \multicolumn{6}{|l|}{ Year of hospitalisation } \\
\hline 2010 & 2640 & 88.6 & 340 & 11.4 & 0.1240 \\
\hline 2011 & 5503 & 89.9 & 621 & 10.1 & \\
\hline 2012 & 6113 & 90.1 & 672 & 9.9 & \\
\hline 2013 & 3349 & 89.3 & 400 & 10.7 & \\
\hline \multicolumn{6}{|l|}{ Age (years) } \\
\hline$<65$ & 8898 & 96.6 & 312 & 3.4 & $<0.0001$ \\
\hline$\geq 65$ & 8707 & 83.5 & 1721 & 16.5 & \\
\hline \multicolumn{6}{|l|}{ Hospital-level variables } \\
\hline \multicolumn{6}{|l|}{ Hospital volume } \\
\hline 1st quartile (<368 cases; 92 hospitals) & 3950 & 82.2 & 858 & 17.8 & $<0.0001$ \\
\hline 2nd quartile (368-604 cases; 10 hospitals) & 3721 & 91.1 & 363 & 8.9 & \\
\hline 3rd quartile (605-1213 cases; 8 hospitals) & 4600 & 92.1 & 394 & 7.9 & \\
\hline 4th quartile ( $\geq 1214$ cases $\geq ; 4$ hospitals) & 5334 & 92.7 & 418 & 7.3 & \\
\hline \multicolumn{6}{|l|}{ Specialist proportion } \\
\hline Below median ( $<58.62 \% ; 18$ hospitals) & 9094 & 91.6 & 835 & 8.4 & $<0.0001$ \\
\hline Above median ( $\geq 58.62 \% ; 96$ hospitals) & 8511 & 87.7 & 1198 & 12.3 & \\
\hline \multicolumn{6}{|l|}{ Number of cardiothoracic medicine specialists } \\
\hline Below median ( $<3 ; 98$ hospitals) & 7678 & 86.7 & 1181 & 13.3 & $<0.0001$ \\
\hline Above median ( $\geq 3 ; 16$ hospitals) & 9927 & 92.1 & 852 & 7.9 & \\
\hline \multicolumn{6}{|l|}{ Number of emergency medicine specialists } \\
\hline Below median ( $<5 ; 95$ hospitals) & 7590 & 86.3 & 1206 & 13.7 & $<0.0001$ \\
\hline Above median ( $\geq 5 ; 19$ hospitals) & 10015 & 92.4 & 827 & 7.6 & \\
\hline \multicolumn{6}{|l|}{ Number of beds } \\
\hline Below median ( $<617 ; 100$ hospitals) & 7768 & 86.4 & 1227 & 13.6 & $<0.0001$ \\
\hline Above median ( $\geq 617 ; 14$ hospitals) & 9837 & 92.4 & 806 & 7.6 & \\
\hline Number of ICU beds & & & & & \\
\hline Below median (<49; 101 hospitals) & 7925 & 86.2 & 1271 & 13.8 & $<0.0001$ \\
\hline Above median ( $\geq 49 ; 13$ hospitals) & 9680 & 92.7 & 762 & 7.3 & \\
\hline Number of ER beds & & & & & \\
\hline Below median (<30; 99 hospitals) & 7785 & 86.2 & 1247 & 13.8 & $<0.0001$ \\
\hline Above median ( $\geq 30 ; 15$ hospitals) & 9820 & 92.6 & 786 & 7.4 & \\
\hline Teaching status & & & & & \\
\hline Non-teaching hospital (71 hospitals) & 15475 & 91.2 & 1491 & 8.8 & $<0.0001$ \\
\hline Teaching hospital (43 hospitals) & 2130 & 79.7 & 542 & 20.3 & \\
\hline Ownership & & & & & \\
\hline Public (31 hospitals) & 1145 & 79.2 & 301 & 20.8 & $<0.0001$ \\
\hline Private ( 83 hospitals) & 16460 & 90.5 & 1732 & 9.5 & \\
\hline
\end{tabular}


Table 1 Continued

\begin{tabular}{|c|c|c|c|c|c|}
\hline \multirow[b]{2}{*}{ Variables } & \multicolumn{2}{|c|}{ Survived } & \multicolumn{2}{|l|}{ Died } & \multirow[b]{2}{*}{ p Value } \\
\hline & $\overline{\mathbf{N}}$ & Per cent & $\overline{\mathbf{N}}$ & Per cent & \\
\hline \multicolumn{6}{|l|}{ Region } \\
\hline Metropolitan (36 hospitals) & 6109 & 88.3 & 810 & 11.7 & $<0.0001$ \\
\hline Others (78 hospitals) & 11496 & 90.4 & 1223 & 9.6 & \\
\hline Total & 17605 & 89.5 & 2033 & 10.5 & \\
\hline
\end{tabular}

AMI, acute myocardial infarction; ER, emergency room; ICU, intensive care unit; NHI, National Health Insurance.

mortality in only the below median group. However, there were no statistically significant differences in the above median group in both analyses (figures 1 and 2).

In the subgroup analysis by regional characteristics, there were differences in the relationship between hospital volume and staffing and 30-day mortality. Hospitalisation cases with a higher AMI volume were associated with a lower risk of 30-day mortality in only non-metropolitan areas. In addition, lower 30-day mortality was associated with a higher proportion of specialists, but this relationship was also statistically significant in only non-metropolitan regions (figure 3).

\section{DISCUSSION}

We analysed the relationships between the 30-day mortality after hospitalisation for AMI and hospital volume, structural characteristics and hospital staffing level to establish which factors affect AMI mortality. Our results indicate that higher AMI volume is associated with better outcomes, most likely because high volume for a specific disease increases expertise. ${ }^{23-26}$ In addition, better outcomes were associated with a greater proportion of specialists and a higher number of emergency medicine physicians. These factors are typically associated with better quality of care. ${ }^{27-29}$ Many previous analyses on hospital volume, structural characteristics, etc have been conducted.

However, our study has several strengths compared with previous studies. First, we conducted a multilevel analysis using national health insurance claim data that reflected inpatient and hospital characteristics. Thus, our models considered the hierarchical nature of claim data, capturing the diversity of inpatients and hospitals as much as possible. Second, our study considered the hospital staffing level, including the proportion of specialists and number of emergency medicine specialists and cardiothoracic specialists. Of course, studies reporting better AMI outcomes in association with the presence of specialists have been published in the past, ${ }^{27} 30$ using cardiologists as a proxy for AMI surgery expertise. ${ }^{31} 32$ However, we included the number of emergency medicine specialists in our analysis, as it is very important to treat inpatients with AMI quickly and most are hospitalised via the ER. ${ }^{33-35}$ Our results emphasise the need for emergency medicine specialists in acute care, suggesting that it is needed to improve not only quantity of hospital staffing but also quality of those for effective care in the management of inpatients with AMI. Unfortunately, hospitals have not yet been able to successfully address the lack of emergency medicine specialists in the current business climate. ${ }^{36-38}$ We also analysed the proportion of specialists in our study, obtaining results similar to those reported in other studies. ${ }^{17}{ }^{39}$ However, our study considered the relative number of specialists rather than the absolute number, using proportion. Collectively, our findings suggest that improvements be made to hospitals' human resource pool, which will create an economic burden and challenge policymakers and hospital managers. Third, we considered hospital characteristics such as ownership and teaching status. Our results, similar to those in previous studies, ${ }^{40}$ indicate that private hospitals have better outcomes. There were some concerns that the differences in outcomes by ownership status might have been affected by differences in the proportion of medical aid beneficiaries due to unmeasured bias. Nevertheless, better quality management in public hospitals seems necessary, though a clear assessment of quality is essential before management changes are initiated. Some hospitals, for example, may appear to have worse outcomes than others simply due to a failure to properly adjust for the inpatient mix. Finally, we performed subgroup analysis by median values for emergency medicine or cardiothoracic medicine specialists in hospital. In the results of the analyses, higher hospital volume was more significantly associated with a lower risk in 30-day mortality in only the below median group for emergency or cardiothoracic medicine specialists. These findings suggest that a higher volume could result in an increase in expertise in managing AMI in hospitals without either emergency or cardiothoracic medicine specialists. Conversely, hiring more specialists in either emergency or cardiothoracic medicine would be an efficient strategy for the management of patients with AMI in hospitals without higher hospital volume. In addition, the results of the subgroup analysis by region indicated a volume-outcome relationship in only non-metropolitan regions, as hospitals in metropolitan regions had already reached sufficient expertise due to the presence of more specialists and sufficient hospital volume of visiting patients.

Our study also has several limitations. First, we only included 156 hospitals in this study, as the national 
Table 2 Factors associated with 30-day mortality after AMI hospitalisation, according to a multilevel model

\begin{tabular}{|c|c|c|c|}
\hline Variables & OR & $95 \% \mathrm{Cl}$ & \\
\hline Inpatient-level variables & & & \\
\hline Major diagnosis & & & \\
\hline Acute transmural myocardial infarction of anterior wall & 1.463 & 1.039 & 2.061 \\
\hline Acute transmural myocardial infarction of inferior wall & 1.000 & - & - \\
\hline Acute transmural myocardial infarction of other sites & 2.032 & 1.062 & 3.887 \\
\hline Acute transmural myocardial infarction of unspecified sites & 2.451 & 1.729 & 3.475 \\
\hline Acute subendocardial myocardial infarction & 1.190 & 0.866 & 1.637 \\
\hline Acute myocardial infarction, unspecified & 1.986 & 1.494 & 2.640 \\
\hline Charlson Comorbidity Index & & & \\
\hline 0 & 1.000 & - & - \\
\hline 1 & 1.045 & 0.657 & 1.663 \\
\hline 2 & 0.985 & 0.621 & 1.562 \\
\hline $3+$ & 2.016 & 1.293 & 3.143 \\
\hline Sex & & & \\
\hline Male & 1.000 & - & - \\
\hline Female & 1.426 & 1.244 & 1.635 \\
\hline Type of insurance coverage & & & \\
\hline $\mathrm{NHI}$ & 1.000 & - & - \\
\hline Medical Aid & 1.222 & 0.998 & 1.497 \\
\hline Age (years) & & & \\
\hline$<65$ & 1.000 & - & - \\
\hline$\geq 65$ & 3.359 & 2.724 & 4.141 \\
\hline Year of hospitalisation & & & \\
\hline 2010 & 1.000 & - & - \\
\hline 2011 & 0.790 & 0.646 & 0.965 \\
\hline 2012 & 0.770 & 0.633 & 0.936 \\
\hline 2013 & 0.813 & 0.654 & 1.011 \\
\hline Hospital-level variables & & & \\
\hline Hospital volume & & & \\
\hline 1st quartile ( $<368$ cases; 92 hospitals) & 1.000 & - & - \\
\hline 2nd quartile (368-604 cases; 10 hospitals) & 0.811 & 0.658 & 0.998 \\
\hline 3rd quartile (605-1213 cases; 8 hospitals) & 0.648 & 0.500 & 0.840 \\
\hline 4th quartile ( $\geq 1214$ cases $\geq ; 4$ hospitals) & 0.807 & 0.573 & 1.138 \\
\hline Specialist proportion & & & \\
\hline Below median ( $<58.62 \% ; 18$ hospitals) & 1.000 & - & - \\
\hline Above median ( $\geq 58.62 \%$; 96 hospitals) & 0.789 & 0.663 & 0.940 \\
\hline Number of cardiothoracic medicine specialists & & & \\
\hline Below median ( $<3$; 98 hospitals) & 1.000 & - & - \\
\hline Above median ( $\geq 3 ; 16$ hospitals) & 1.013 & 0.842 & 1.219 \\
\hline Number of emergency medicine specialists & & & \\
\hline Below median ( $<5 ; 95$ hospitals) & 1.000 & _- & - \\
\hline Above median ( $\geq 5 ; 19$ hospitals) & 0.760 & 0.619 & 0.933 \\
\hline Number of beds & & & \\
\hline Below median ( $<617 ; 100$ hospitals) & 1.000 & - & - \\
\hline Above median ( $\geq 617 ; 14$ hospitals) & 0.882 & 0.677 & 1.151 \\
\hline Number of ICU beds & & & \\
\hline Below median $(<49 ; 101$ hospitals $)$ & 1.000 & _- & _- \\
\hline Above median ( $\geq 49 ; 13$ hospitals) & 1.091 & 0.861 & 1.383 \\
\hline Number of ER beds & & & \\
\hline Below median (<30; 99 hospitals) & 1.000 & - & - \\
\hline Above median ( $>30 ; 15$ hospitals) & 0.862 & 0.693 & 1.073 \\
\hline Teaching status & & & \\
\hline Non-teaching hospital ( 71 hospitals) & 1.000 & _- & _- \\
\hline Teaching hospital ( 43 hospitals) & 0.716 & 0.570 & 0.900 \\
\hline Ownership & & & \\
\hline Public (31 hospitals) & 1.000 & - & - \\
\hline Private ( 83 hospitals) & 0.781 & 0.626 & 0.976 \\
\hline
\end{tabular}


Table 2 Continued

\begin{tabular}{lcc}
\hline Variables & OR & $\mathbf{9 5 \%} \mathbf{C l}$ \\
\hline Region & & - \\
$\quad$ Metropolitan (36 hospitals) & 1.000 & $\mathbf{0 . 7 3 2}$ \\
Others (78 hospitals) & $\mathbf{0 . 8 5 4}$ & $\mathbf{0 . 9 9 5}$ \\
\hline Statistically significant results are shown in bold typeface. & & \\
AMI, acute myocardial infarction; ICU, intensive care unit; NHI, National Health Insurance. &
\end{tabular}

health insurance claim data we used did not include data from all hospitals in South Korea due to access authority and ethical issues. Therefore, we used the propensity score matching for effective data extraction. In using such methodology, there were some concerns related to the process of data extraction. In fact, it might have been better to identify public hospitals that admitted patients with AMI and then randomly sample private hospitals, but we were unable to do so due to regulations in the process of extracting data from the national database. Thus, it may be difficult to generalise our results to South Korea as a whole. Second, we were unable to determine whether the same inpatients were hospitalised multiple times, as the data used in our study were based on only hospitalisation cases, not inpatient details.
The details of whether patients transferred to other hospitals in order to receive better specialised care could not be considered in this study, because those types of information were unavailable in the data used in this study. In addition, details on the procedures used, such as angioplasty, percutaneous coronary intervention and coronary artery bypass graft surgery, were not included in the available data and therefore not considered in our analysis, despite such information being an important indicator of AMI care quality. Third, we were unable to analyse associations between AMI mortality and cardiologist numbers, as the hospital staffing level data used in our study only included the number of cardiothoracic and emergency medicine specialists and not the number of cardiologists. Fourth, the data used in this

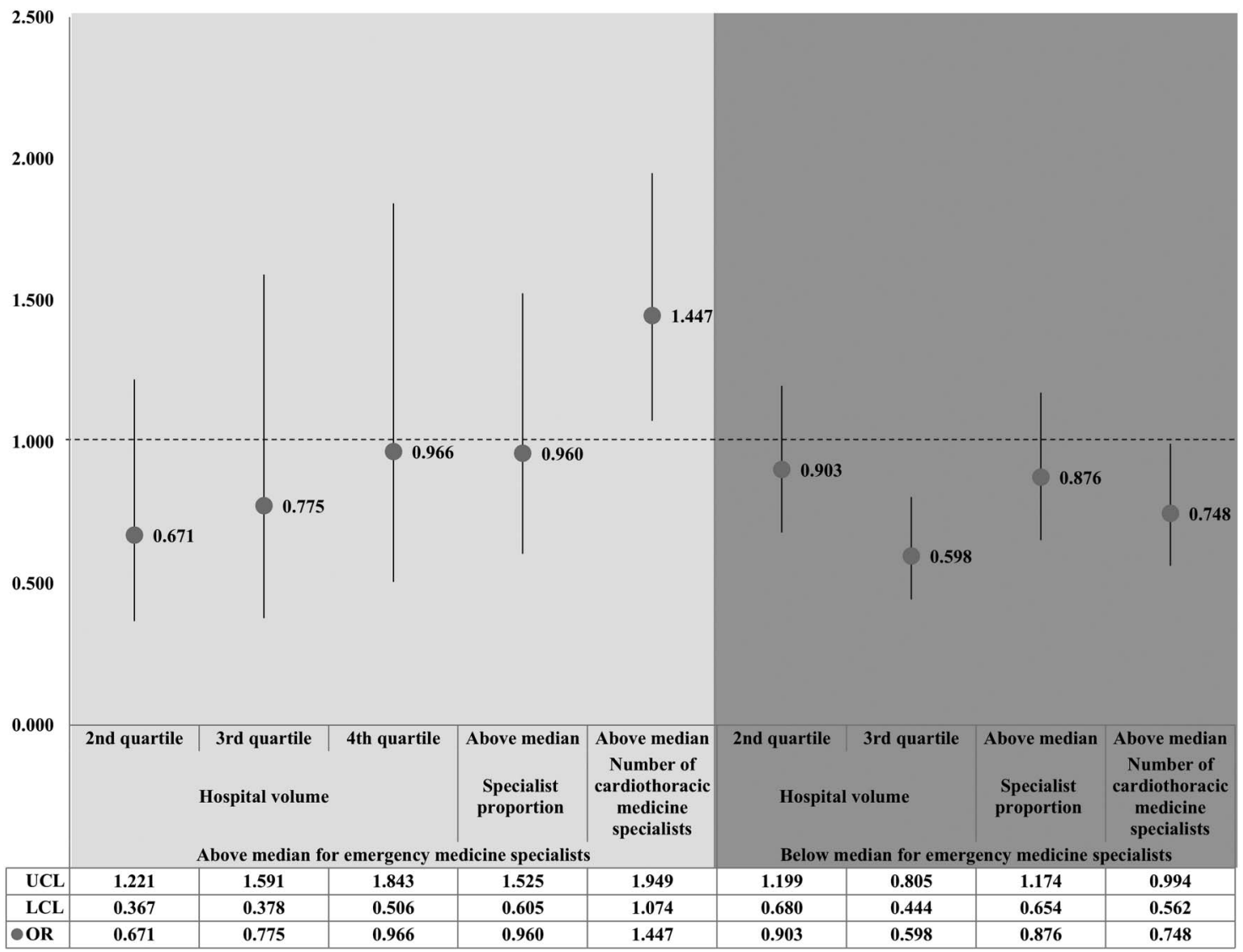

Figure 1 Factors associated with 30-day mortality after hospitalisation, stratified by median value of emergency medicine specialists. The OR as marked as circle point was calculated by multilevel analysis adjusted for inpatient-level characteristics and hospital-level characteristics, and results were statistically significant if each bar as marked to SD is not reached the cutoff line in 1.00. UCL=95\% upper confidence limit, $L C L=95 \%$ lower confidence limit. 


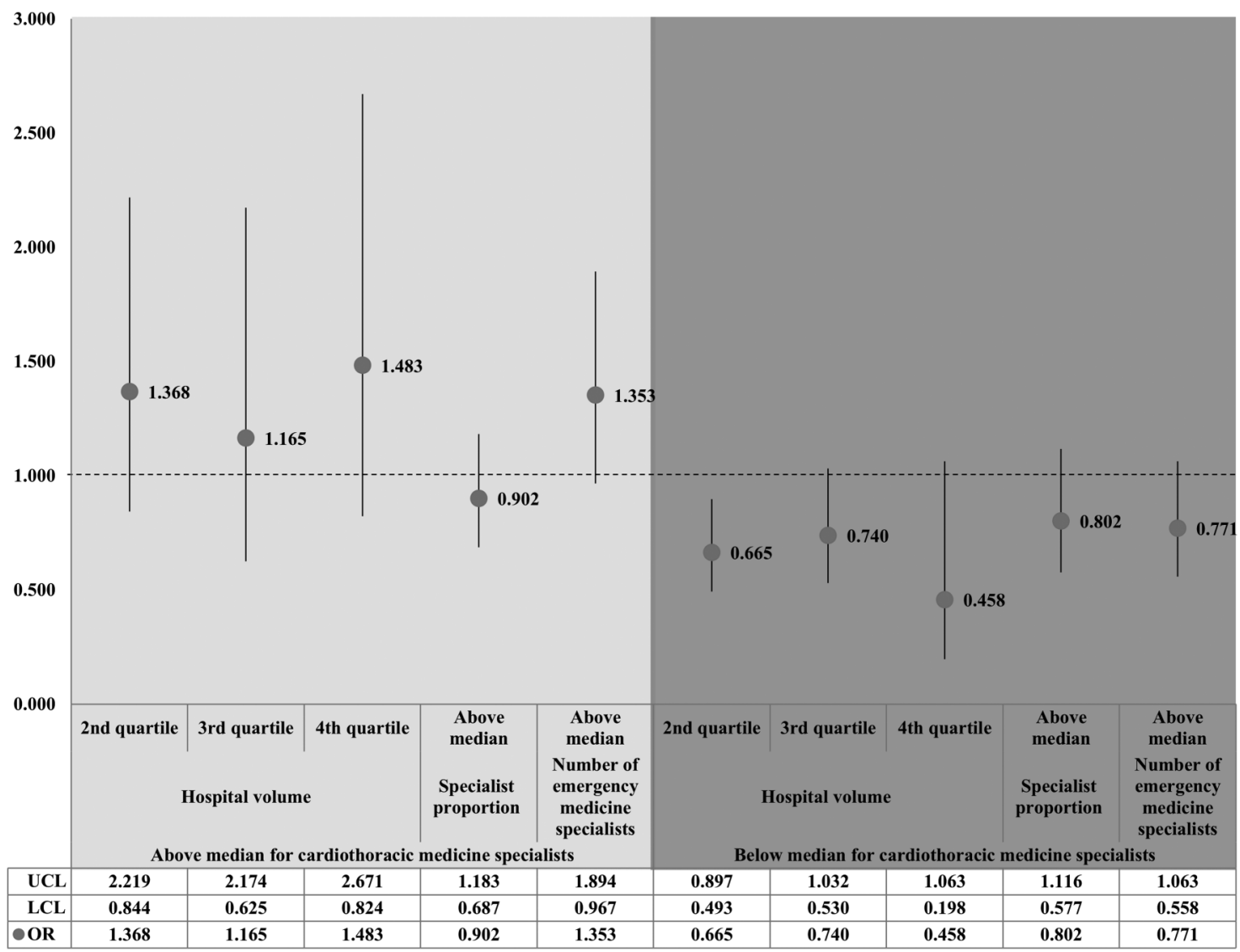

Figure 2 Factors associated with 30-day mortality after hospitalisation, stratified by median value of cardiothoracic medicine specialists. The OR, marked by a circle, was calculated by multilevel analysis adjusted for inpatient-level characteristics and hospital-level characteristics, and results were statistically significant if each bar as marked to SD has not reached the cutoff line in 1.00. $U C L=95 \%$ upper confidence limit, $L C L=95 \%$ lower confidence limit.

study could reflect information about patient-level and hospital-level variables, because these data were collected into the national database by NHIS. However, we could not include all of the detailed factors such as the distance to hospitals due to data limitations. Thus, it may be difficult to make strong conclusions solely on the basis of our results. Additionally, we were unable to determine the difference of the incremental relationship per 1 unit increases by high or low numbers of cardiothoracic or emergency medicine specialists, as the range of data used in our study was too small to perform an analysis. Finally, our study considered neither costs nor readmissions. Therefore, future studies investigating these issues are warranted.

Despite these limitations, our findings suggest that inpatient-level and hospital-level factors have a substantial impact on 30-day mortality after hospitalisation for AMI, providing valuable information for health policymakers. In 2007, the South Korea government introduced a programme designed to improve the management of patients with AMI by assessing performance based on rates of reperfusion treatment, medication and survival. On the basis of the results of this assessment, the government provided incentives or disincentives to hospitals. Although many previous studies determined that higher hospital volume was associated with better outcomes in the management of patients with AMI, the programme did not reflect structural characteristics including hospital staffing and hospital volume in evaluating the performance of each hospital. Given that hospitals with a higher volume or a higher proportion of specialists achieved better AMI care outcomes in the results of our study, health policymakers need to consider adding such indicators in performance evaluation tools used in management programmes. Otherwise, health policymakers must consider increasing the proportion of specialists in hospitals, which would include regulating the hiring of emergency medicine specialists. In conclusion, health policymakers and hospital managers must make an effort to reduce avoidable AMI deaths based on such considerations.

\section{CONCLUSIONS}

Outcomes for AMI care were better in hospitals with either a higher AMI volume or a higher proportion of specialists. Specifically, hospitals that employed more emergency medicine specialists had better AMI survival rates. In addition, the reduction of risk for 30-day mortality was better associated with the median value for 


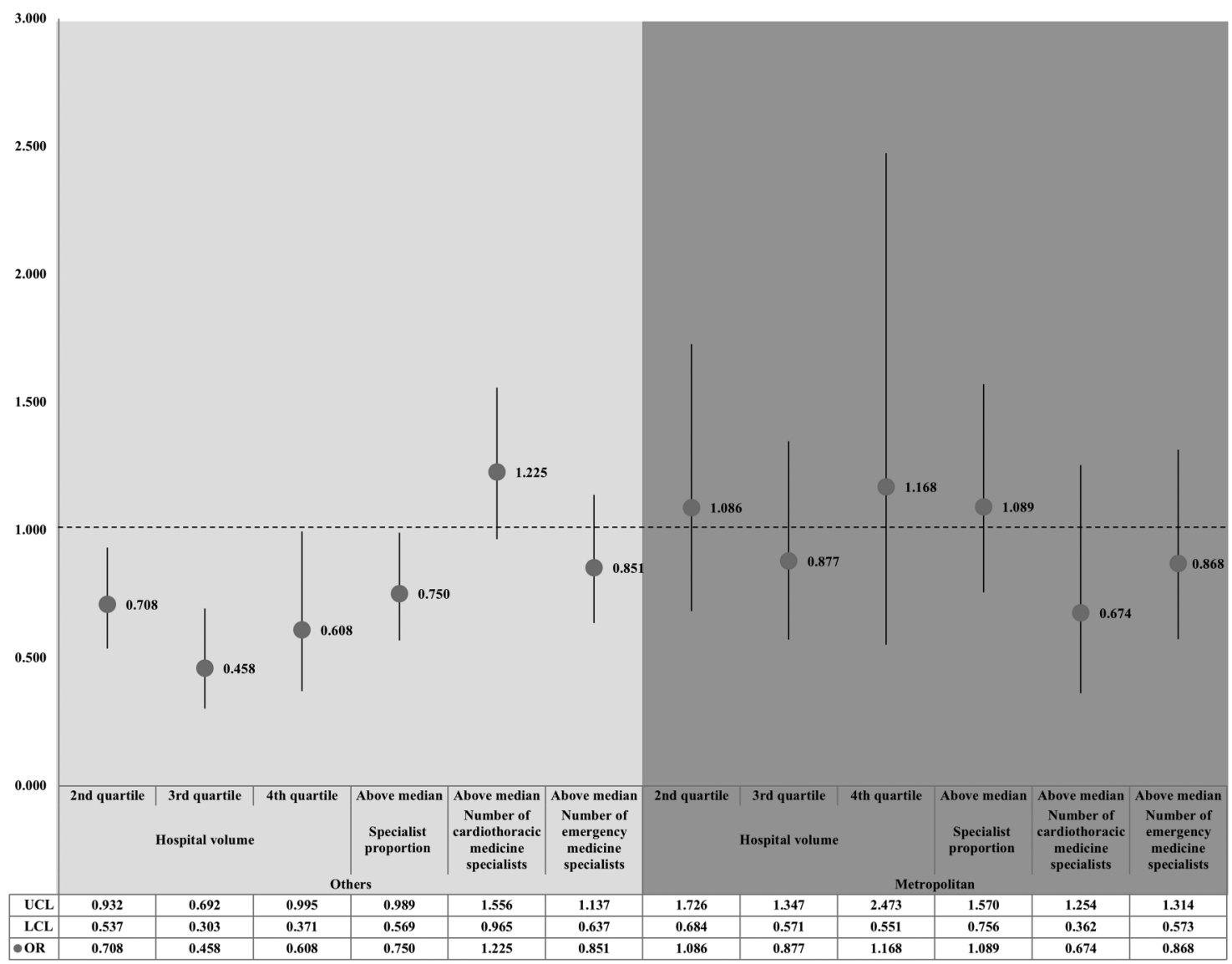

Figure 3 Factors associated with 30-day mortality after hospitalisation, stratified by regional hospital characteristics of hospital. The OR, as marked by a circle, was calculated by multilevel analysis adjusted for inpatient-level characteristics and hospital-level characteristics, and results were statistically significant if each bar as marked to SD has not reached the cutoff line in 1.00. UCL=95\% upper confidence limit, $\mathrm{LCL}=95 \%$ lower confidence limit.

emergency or cardiothoracic medicine specialists, respectively. These findings are likely to be helpful in establishing a management strategy for patients with AMI. Given that hospitals with higher volumes or a higher proportion of specialists achieved better AMI care outcomes in the results of our study, health policymakers need to consider adding such indicators in performance evaluation tools used in management programmes when creating effective alternative strategies targeting to reduce avoidable deaths due to AMI.

\section{Author affiliations}

${ }^{1}$ Department of Public Health, Graduate School, Yonsei University, Seoul, Republic of Korea

${ }^{2}$ Institute of Health Services Research, Yonsei University College of Medicine, Seoul, Republic of Korea

${ }^{3}$ Department of Health Administration and Management, Soonchunhyang University, Asan, Republic of Korea

${ }^{4}$ Department of Preventive Medicine, Yonsei University College of Medicine, Seoul, Republic of Korea

${ }^{5}$ Department of Hospital Management, Eulji University, Seongnam, Republic of Korea

Contributors $\mathrm{K}-\mathrm{TH}$ designed the study, researched the data, performed the statistical analyses and wrote the manuscript. SJK, S-IJ, K-BY, SYL and E-CP contributed to the discussion and reviewed and edited the manuscript. E-CP is the guarantor of this work and, as such, had full access to all the data in the study and takes responsibility for the integrity of the data and the accuracy of the data analysis. The professional English-language scientific editing staff at BioScience Writers, LLC (http://www.biosciencewriters.com/ verify; validation code: 3E6C2C5E-43DA-40E7-85B9-DC07299ABBA0) provided English translation services and scientific editing. In addition, WK provided re-editing services for our manuscript to improve the quality of scientific writing.

Funding This research received no specific grant from any funding agency in the public, commercial or not-for-profit sectors.

Competing interests None declared.

Provenance and peer review Not commissioned; externally peer reviewed.

Data sharing statement No additional data are available.

Open Access This is an Open Access article distributed in accordance with the Creative Commons Attribution Non Commercial (CC BY-NC 4.0) license, which permits others to distribute, remix, adapt, build upon this work noncommercially, and license their derivative works on different terms, provided the original work is properly cited and the use is non-commercial. See: http:// creativecommons.org/licenses/by-nc/4.0/

\section{REFERENCES}

1. Boersma E, Mercado N, Poldermans D, et al. Acute myocardial infarction. Lancet 2003;361:847-58.

2. Schiele F, Meneveau N, Seronde MF, et al. Compliance with guidelines and 1-year mortality in patients with acute myocardial infarction: a prospective study. Eur Heart J 2005;26:873-80. 
3. Eagle KA, Montoye CK, Riba AL, et al. Guideline-based standardized care is associated with substantially lower mortality in medicare patients with acute myocardial infarction: the American College of Cardiology's Guidelines Applied in Practice (GAP) Projects in Michigan. J Am Coll Cardiol 2005;46:1242-8.

4. Health Insurance Review \& Assessment Service. Result of evaluating the acute care quality for acute myocardial infarction, 2013.

5. Organization for Economic Cooperation and Development. Health at a Glance 2013: OECD Indicators. 2013.

6. World Health Organization. Global health risks: mortality and burden of disease attributable to selected major risks. 2009.

7. Thiemann DR, Coresh J, Oetgen WJ, et al. The association between hospital volume and survival after acute myocardial infarction in elderly patients. N Engl J Med 1999;340:1640-8.

8. Magid DJ, Calonge BN, Rumsfeld JS, et al. Relation between hospital primary angioplasty volume and mortality for patients with acute MI treated with primary angioplasty vs thrombolytic therapy. JAMA 2000;284:3131-8.

9. Krumholz HM, Wang Y, Mattera JA, et al. An administrative claims model suitable for profiling hospital performance based on 30-day mortality rates among patients with an acute myocardial infarction. Circulation 2006;113:1683-92.

10. Krumholz HM, Merrill AR, Schone EM, et al. Patterns of hospital performance in acute myocardial infarction and heart failure 30-day mortality and readmission. Circ Cardiovasc Qual Outcomes 2009;2:407-13.

11. Kaneko T, Hirakawa K, Fushimi K. Relationship between peri-operative outcomes and hospital surgical volume of total hip arthroplasty in Japan. Health Policy 2014;117:48-53.

12. Lee KS, Kwak JM. Effect of patient risk on the volume-outcome relationship in obstetric delivery services. Health Policy 2014;118:407-12.

13. Lee KL, Woodlief LH, Topol EJ, et al. Predictors of 30-Day Mortality in the Era of Reperfusion for Acute Myocardial Infarction Results From an International Trial of 41021 Patients. Circulation 1995;91:1659-68.

14. Gan SC, Beaver SK, Houck PM, et al. Treatment of acute myocardial infarction and 30-day mortality among women and men. N Engl J Med 2000;343:8-15.

15. Chen J, Radford MJ, Wang Y, et al. Do "America's Best Hospitals" perform better for acute myocardial infarction?. $N$ Engl J Med 1999;340:286-92.

16. Alter DA, Naylor CD, Austin P, et al. Effects of socioeconomic status on access to invasive cardiac procedures and on mortality after acute myocardial infarction. N Engl J Med 1999;341:1359-67.

17. Jollis JG, DeLong ER, Peterson ED, et al. Outcome of acute myocardial infarction according to the specialty of the admitting physician. N Engl J Med 1996;335:1880-7.

18. Häkkinen $\mathrm{U}$, Rosenqvist $\mathrm{G}$, Peltola $\mathrm{M}$, et al. Quality, cost, and their trade-off in treating AMI and stroke patients in European hospitals. Health Policy 2014;117:15-27.

19. Park S, Lee J, Ikai H, et al. Quality of care and in-hospital resource use in acute myocardial infarction: evidence from Japan. Health Policy 2013;111:264-72.

20. Schreyögg J, Stargardt T, Tiemann O. Costs and quality of hospitals in different health care systems: a multi-level approach with propensity score matching. Health Econ 2011;20:85-100.

21. Rubin DB, Thomas N. Combining propensity score matching with additional adjustments for prognostic covariates. J Am Stat Assoc 2000;95:573-85.
22. Kwon S. Thirty years of national health insurance in South Korea: lessons for achieving universal health care coverage. Health Policy Plan 2009;24:63-71.

23. Chen EW, Canto JG, Parsons LS, et al. Relation between hospital intra-aortic balloon counterpulsation volume and mortality in acute myocardial infarction complicated by cardiogenic shock. Circulation 2003:108:951-7.

24. Sollano JA, Gelijns AC, Moskowitz AJ, et al. Volume-outcome relationships in cardiovascular operations: New York State, 19901995. J Thorac Cardiovasc Surg 1999;117:419-30.

25. Garnick DW, DeLong ER, Luft HS. Measuring hospital mortality rates: are 30-day data enough? Ischemic Heart Disease Patient Outcomes Research Team. Health Serv Res 1995;29:679.

26. Vakili BA, Kaplan R, Brown DL. Volume-outcome relation for physicians and hospitals performing angioplasty for acute myocardial infarction in New York state. Circulation 2001;104:2171-6.

27. Starfield B, Shi L, Grover A, et al. The effects of specialist supply on populations' health: assessing the evidence. Cancer 2005;103:23.18.

28. Diette GB, Skinner EA, Nguyen TT, et al. Comparison of quality of care by specialist and generalist physicians as usual source of asthma care for children. Pediatrics 2001;108:432-7.

29. Fisher ES, Wennberg JE. Health care quality, geographic variations, and the challenge of supply-sensitive care. Perspect Biol Med 2003:46:69-79.

30. Park S, Lee J, Ikai $\mathrm{H}$, et al. Decentralization and centralization of healthcare resources: investigating the associations of hospital competition and number of cardiologists per hospital with mortality and resource utilization in Japan. Health Policy 2013;113 $100-9$.

31. Casale PN, Jones JL, Wolf FE, et al. Patients treated by cardiologists have a lower in-hospital mortality for acute myocardial infarction. J Am Coll Cardiol 1998;32:885-9.

32. Tu JV, Austin PC, Chan BT. Relationship between annual volume of patients treated by admitting physician and mortality after acute myocardial infarction. JAMA 2001;285:3116-22.

33. Lee TH, Rouan GW, Weisberg MC, et al. Clinical characteristics and natural history of patients with acute myocardial infarction sent home from the emergency room. Am J Cardiol 1987;60:219-24.

34. Schull MJ, Vermeulen M, Slaughter G, et al. Emergency department crowding and thrombolysis delays in acute myocardial infarction. Ann Emerg Med 2004;44:577-85.

35. Lee TH, Cook EF, Weisberg M, et al. Acute chest pain in the emergency room: identification and examination of low-risk patients. Arch Intern Med 1985;145:65-9.

36. Cooper RA. There's a shortage of specialists: is anyone listening? Acad Med 2002;77:761-6.

37. McConnell KJ, Johnson LA, Arab N, et al. The on-call crisis: a statewide assessment of the costs of providing on-call specialist coverage. Ann Emerg Med 2007;49:727-33.e1-18.

38. Kellermann AL. Crisis in the emergency department. N Engl J Med 2006;355:1300-3.

39. Ayanian JZ, Guadagnoli E, McNeil BJ, et al. Treatment and outcomes of acute myocardial infarction among patients of cardiologists and generalist physicians. Arch Intern Med 1997;157:2570-6.

40. Jensen PH, Webster E, Witt J. Hospital type and patient outcomes: an empirical examination using AMI readmission and mortality records. Health Econ 2009;18:1440-60. 